\title{
IgG isotypic antibodies to crude Plasmodium falciparum blood-stage antigen associated with placental malaria infection in parturient Cameroonian women.
}

\author{
Judith K Anchang-Kimbi ${ }^{1}$, Eric Akum Achidi ${ }^{2}$, Blaise Nkegoum ${ }^{3}$, Joseph-Marie N Mendimi ${ }^{4}$, \\ Eva Sverremark-Ekström ${ }^{5}$, Marita Troye-Blomberg ${ }^{6}$
}

1. Department of Zoology and Animal Physiology, University of Buea, Buea 63.

2. Department of Biochemistry and Molecular Biology, University of Buea, Buea-63, Cameroon.

3. Department of Anatomy and Pathology, University of Yaoundé Teaching Hospital, Yaoundé-812.

4. Department of Anatomy and Pathology, University of Yaoundé I Teaching Hospital, Yaoundé-812.

5. Department of Molecular Bioscience, Wenner-Gren Institute, Stockholm University, SE-10691 Stockholm.

6. Department of Molecular Bioscience, Wenner-Gren Institute, Stockholm University, SE- 10691 Stockholm.

\begin{abstract}
Background: Few studies have reported an association between placental malaria (PM) infection and levels of isotypic antibodies against non-pregnancy associated antigens.

Objective: To determine and evaluate IgG isotypic antibody levels to crude P. falciparum blood stage in women with and without PM infection.

Methods: Levels of IgG (IgG1-IgG4) and IgM to crude P. falciparum blood stage antigen were measured by ELISA in 271 parturient women. Placental malaria infection was determined by placental blood microscopy and placental histology. Age, parity and intermittent preventive treatment during pregnancy with sulphadoxine-pyrimethamine (IPTp-SP) usage were considered during analysis.

Results: P. falciparum-specific IgG1 (96.5\%) and IgG3 (96.7\%) antibodies were predominant compared with IgG2 (64.6\%) and IgG4 (49.1\%). Active PM infection was associated with significant increased levels of IgG1, IgG4 and IgM while lower levels of these antibodies were associated with uptake of two or more IPTp-SP doses. PM infection was the only independent factor associated with IgG4 levels. Mean IgG1 + IgG3/IgG2 + IgG4 and IgG1 + IgG2 +IgG3/ IgG4 ratios were higher among the PM-uninfected group while $\mathrm{IgG} 4 / \mathrm{IgG} 2$ ratio prevailed in the infected group.

Conclusion: PM infection and IPTp-SP dosage influenced P. falciparum-specific isotypic antibody responses to blood stage antigens. An increase in IgG4 levels in response to PM infection is of particular interest.

Keywords: Placental malaria infection, isotypic antibodies, crude Plasmodium falciparum antigen.

DOI: http://dx.doi.org/10.4314/ahs.v16i4.17

Cite as: Anchang-Kimbi JK, Achidi EA, Nkegoum B, Mendimi J-MN, Sverremark-Ekström E, Troye-Blomberg M. IgG isotypic antibodies to crude Plasmodium falciparum blood-stage antigen associated with placental malaria infection in parturient Cameroonian women. Afri Health $S_{c i}$ 2016;16(4): 1007-1017. bttp://dx.doi.org/10.4314/abs.v16i4.17
\end{abstract}

\section{Introduction}

Women are at higher risk of malaria infection and disease when pregnant ${ }^{1}$. This susceptibility may represent a combination of altered immune and hormonal function coupled with the unique ability of infected erythrocytes (IEs) to sequester in the placenta through adhesion to chondroitin sulfate A (CSA) ${ }^{2}$. It has been suggested that

\section{Corresponding author: \\ Judith K Anchang-Kimbi, \\ Department of Zoology and Animal \\ Physiology, University of Buea, \\ Buea 63, Cameroon. \\ Email:kuoh2000@yahoo.fr}

the massive accumulation of Plasmodium falciparum-IEs in the intervillous spaces (IVS) of the placenta triggers the deleterious effects of malaria in pregnant women and their offspring ${ }^{3}$. The decreasing risk of malaria with subsequent pregnancies is attributed to parity-dependent acquisition of antibodies against these placental parasites, which has been associated with lower risks of placental parasitemia $^{4,5}$, maternal anaemia ${ }^{6}$ and low birth weight ${ }^{7}$. In addition to the parity-specific immunity, age-associated immunity has also been suggested to play an important part in the control of infection during pregnancy in areas of high and stable transmission ${ }^{8}$.

Recent findings show that PM consists of parasites expressing var genes other than var2csa that can stimulate 
the production of antibodies against a wide range of parasite antigens ${ }^{9,10}$. Thus placental P. falciparum infection has been associated with increased IgG levels against merozoite antigens and parasite isolates from pregnant and non-pregnant hosts ${ }^{10}$. Furthermore antibodies against P. falciparum antigens not specifically associated with pregnancy have also been shown to increase with parity ${ }^{10-12}$.

Studies have shown that the responses for both non-VSAPAM $^{13,14}$ and VSAPAM ${ }^{10-15}$ are dominated by the cytophilic subclass IgG1, followed by IgG3 consistent with data obtained from non-pregnant adults and children ${ }^{14,16,17}$. The predominance of IgG1 and IgG3 cytophilic antibody in endemic areas has been associated with either lower parasitaemia ${ }^{18}$ or a lower risk of malaria attack ${ }^{19}$. Similarly, levels of IgG1 and IgG3 have been shown to correlate with the ability of plasma from pregnant women to inhibit parasite adhesion to CSA (Chondriotin Sulphate A) in vitro 15 suggesting that these antibodies may function by blocking parasite adhesion to placental CSA. Conversely, high levels of IgG2, a non-cytophilic antibody have been shown to be associated with low risk of acquiring P. falciparum infection ${ }^{20,21}$ in individuals carrying a specific allelic variant of monocytes Fc $\gamma$ RIIA receptor that can bind $\operatorname{IgG} 2^{21}$, whereas, high IgG4 levels been associated with high risk of infection and malaria attack by blocking protective mechanisms individuals living in endemic areas ${ }^{21}$.

A recent study in a peri-urban setting in Uganda showed that naturally acquired immunity to merozoite surface antigens (MSP19 and MSP42) and serine repeat antigen-5(SE36) in pregnant women were associated with reduced placental parasitemia ${ }^{22}$. We have previously shown that plasma from Cameroonian parturient mothers resident in the South West Region possess inhibitory antibodies that are involved with blocking the re-invasion of host red blood cells by erythrocytic merozoites in vitro ${ }^{23}$. Thus, in this study we measured and determined IgG (IgG1-G4) isotypic antibody response pattern to a crude P. falciparum blood stage antigen in parturient mothers resident in the same area. Secondly, we investigated if isotypic antibodies play a role in PM infection taking into consideration the effect of maternal age, parity and use of IPTp-SP.

\section{Material and methods}

\section{Ethics statement}

Ethical clearance for the study was obtained from the
South West Regional Delegation of Public Health. Approval to conduct the study in the designated health centre was obtained from Mutengene Health District Medical Officer. Expectant mothers in their third trimester, who fulfilled the specific inclusion criteria and volunteered to participate after adequate sensitisation on the project objectives, methods and possible benefits/risks, were enrolled into the study. Study participants were enrolled if they gave a written informed consent.

\section{Study area and population}

This study was part of the work conducted at the Government Health Centre in the Mutengene Health Area, Mt Area Cameroon region, South west Region, Cameroon from March to October 2007. The characteristics of the study area have been described elsewhere ${ }^{24}$. In brief, malaria transmission is perennial, with some seasonality. Intermittent preventive treatment during pregnancy (IPTp) consists of the use of regular treatment doses of $\mathrm{SP}$ as stipulated by $\mathrm{WHO}^{25}$. SP dosage and compliance were confirmed from patient-held ANC cards, patient's medical record book and by personal interview. The prevalence of HIV infection among the women was $5.6 \%{ }^{24}$. Mother's age, parity status and gestational age were recorded.

\section{Sample collection and processing}

Maternal peripheral venous blood $(2 \mathrm{ml})$ was collected within 24 hours after delivery from parturient women who consented to participate in the study for antibody analysis. Immediately after delivery, the placenta was obtained and a small piece of tissue $(0.5 \mathrm{~cm} 3)$ excised from the centre of the placenta to prepare impression smears. A larger (2 $\mathrm{cm}$ long, $2 \mathrm{~cm}$ wide and $1 \mathrm{~cm}$ thick) biopsy specimen was fixed in 10\% neutral buffered formalin for histopathological assessment as described elsewhere ${ }^{24}$.

\section{Parasitological examination}

Placental impression smears were stained with Giemsa (Sigma) and examined by light microscopy. Placental tissue sections were processed, stained with haematoxylin and eosin and examined as described elsewhere ${ }^{24,26,27}$. Placental malaria parasitaemia and intervillous space monocyte/macrophage count were determined as reported by Ismail et $\mathrm{al}^{27}$ and Rogerson et $\mathrm{al}^{28}$ respectively.

\section{Antibody measurement}

P. falciparum specific- IgG/subclasses (IgG1-4) and IgM 
antibody levels in maternal plasma samples were measured by indirect Enzyme-linked Immunosorbent Assays (ELISA) using crude blood stage parasite extract as capture antigen. Standard curves were obtained in a sandwich ELISA with six dilutions of myeloma proteins of IgG1-4 subclasses and for total anti-malarial IgG and IgM antibodies, with highly purified IgG and IgM respectively. ELISA for the determination of anti-malaria antibodies was carried out using the methods described by Troye-Blomberg et al. ${ }^{29}$ and Perlmann et al. ${ }^{30}$ with some modifications ${ }^{31}$.

Antigen preparation: The F32 strain of P. falciparum was maintained in continuous culture as described by Jensen and Trager (1978) and kept synchronized by repeated treatment with sorbitol. When parasitaemia was $10 \%$ or more with over $70 \%$ of the parasites at the schizont stage, late stage parasites were isolated on $60 \%$ percoll and sonicated to yield the crude P. falciparum antigen (Malaria antigen $(\mathrm{MA})^{32}$. This preparation was used at a concentration of $10 \mu \mathrm{g} / \mathrm{ml}$.

ELISA: Ninety-six-well ELISA plates (Costar, Cambridge, MA, USA) were coated with MA and capture antibodies in sodium carbonate buffer ( $\mathrm{pH}$ 9.6) (50 $\mu \mathrm{l} /$ well) and incubated overnight at $4 \mathrm{oC}$. The optimal concentration for coating the crude blood stage antigen was $10 \mu \mathrm{g} /$ $\mathrm{ml}$ for all isotypes/classes. As capture antibodies for human immunoglobulins (standards), plates were coated with goat anti-human IgG (goat- $\alpha$-huIgG) (Jackson ImmunoResearch Laboratories, Sweden) for IgG, IgG1 and IgG2; mouse- $\alpha$-huIgG (BD Biosciences Pharmingen, USA) for IgG3 and IgG4; and goat- $\alpha$-huIgM (Jackson ImmunoResearch Laboratories, Sweden).

After blocking at $37^{\circ} \mathrm{C}$ with $100 \mu \mathrm{l} /$ well of carbonate buffer containing $0.5 \%$ Bovine serum albumin (BSA) (w/v) for 2 hours, plates were washed four times with ELISA washing buffer $(20 \times)$ solution (phosphate-buffered $\mathrm{NaCl}$ + Tween $20+0.15 \%$ Kathon) (Mabtech, Sweden). The test sera and controls were diluted in incubation buffer (PBS $+20 \% \mathrm{NaN3}+$ Tween $+0.5 \%$ BSA) as follows: for determination of antigen-specific $\operatorname{IgG}(1: 1000), \operatorname{IgG}$ subclasses (1:20 for IgG2 and IgG4, 1:400 for IgG1 and IgG3) and IgM (1:500). Plasma dilutions were added in duplicates and incubated for 1 hour at $37^{\circ} \mathrm{C}$.

Bound IgG and IgM antibodies were detected with alka- line phosphatase conjugated to goat- $\alpha$-huIgG-ALP and goat- $\alpha$-huIgM-ALP respectively (1:2000) (Mabtech, Sweden). IgG subclasses were detected with their respective mouse anti-human; IgG1 (1:1000) (SkyBio, Bedfordshire, UK), IgG2 (1:3000) (Pharmingen, Erembodegem, Belgium), IgG3 (1:1000) (Caltag laboratories,Paisley, UK), IgG4 (1:2000) (Sigma, St Louis, USA). Mouse anti-human IgG2-G4 antibodies were conjugated to biotin. ALP conjugated to streptavidin (Mabtech, Sweden) (1:2000) was added to enhance enzyme bound for IgG2-G4 while ALP- conjugated goat anti-mouse IgG (Dakropatts, Glostrup, Denmark) was used for IgG1 (1:1000). The plates were developed with para-nitrophenyl phosphate (pNPP) (Sigma-Aldrich, USA) as substrate and optical densities (OD) were read at 405nm using the VmaxTM Kinetic microplate reader (Menlo Park, USA).

Concentrations of anti-malarial antibodies were calculated from standard curves obtained in a sandwich ELISA with six dilutions of myeloma (whole molecule) proteins of IgG1-4 isotypes (Biogenesis, Poole, England) and for total anti-malarial $\operatorname{IgG}$ and $\operatorname{IgM}$ antibodies with highly purified IgG and IgM (Jackson ImmunoResearch Laboratories, USA) respectively. Seropositivity was based on mean antibody levels $(\mu \mathrm{g} / \mathrm{ml})+2 \mathrm{SD}$ of 6 non-malaria exposed Swedish donors. The results were expressed and deduced from log-log correlative coefficient of the standard curve.

\section{Definitions and statistical methods}

Data were analysed using SPSS version 17. Age and parity was categorized as follows: age $(\leq 20,21-25,>25)$ years; parity (primiparae, secundiparae and multiparae $(\geq 3$ pregnancies). The number of doses of IPTp-SP prescribed at the ANC was defined as; no IPTp-SP, 1 dose and $\geq 2$ doses. Placental malaria infection was defined as the presence of parasites and/or pigment detected by placental blood microscopy and placental histologic examination and thus classify as active, past and no infection. Placental malaria parasitisation and placental IVS monocyte/macrophage counts were expressed as percentages per 1000 IVS cells.

Antibody concentrations were log transformed and tested for normality using one-Sample Kolmogorov Smirnov test before analyses. We applied a logarithmic transformation based on $2+\log$ (Ig isotype) to allow for zero. The distribution of P. falciparum specific IgG departed

African Health Sciences Vol 16 Issue 4, December, 2016 
significantly from normality in several cases, particularly with respect to total IgG, IgG1 and IgG3. Thus, differences in mean antibody levels between group variables were evaluated by Mann-Whitney rank sum test (MW). Normally distributed antibodies (IgG2, IgG4 and IgM) were compared between groups using independent-sample t-test. Correlations were assessed by Spearman rank correlation coefficient (r). Several exploratory multilinear regression (MLR) (enter) models were run with each antibody isotype as the dependent variable to examine the influence of parity, PM infection and IPTp-SP on antibody levels. Statistical significance was set at $\mathrm{p}<0.05$.

\section{Results}

\section{Characteristics of the study participants}

A total of 271 parturient women were enrolled consecutively into the study. The characteristics of the study pop- ulation are shown in Table 1. Primiparous mothers (19.7 \pm 2.5 years $)$ were significantly younger $(\mathrm{p}<0.001)$ than multiparous mothers $(25.7 \pm 4.4$ years $)$. There were no differences in SP doses received during pregnancy among women of the different parity groups. Thirty-one percent (66/213) of mothers were identified with placental IVS macrophage/monocyte infiltration with a mean monocyte/macrophage count of $0.21 \%$ (range: $0.05-11.5$ ). PM infection was significantly $(\mathrm{p}<0.001)$ associated $(\mathrm{OR}=39.69$; 95\%CI: 9.43-167.07) with the presence of monocyte/macrophage in the placental IVS.

Maternal isotypic antibody seropositivity rates and levels The most prominent differences in proportions among IgG subclasses were seen for P. falciparum-specific IgG1 and $\operatorname{IgG} 3$. The seropositivity rates varied from $49.1 \%$ for $\operatorname{IgG} 4$, to $64.6 \%$ for $\operatorname{IgG} 2$, to $96.5 \%$ for $\operatorname{IgG} 1$ and $96.7 \%$ for IgG3 (Figure 1).

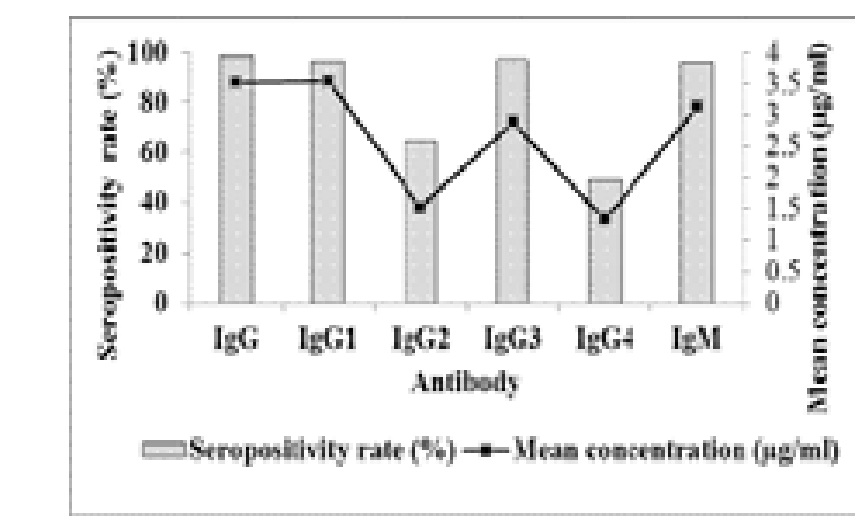

Figure 1: Distribution of maternal $P$. falciparum -specific isotypic antibody seropositivity rates and levels in plasma from 271 Cameroonian parturient women

Generally, isotypic antibody positivity rates were comparable among age, parity and IPTp-SP dosage groups. Higher mean titres of IgG1 and IgG3 compared to IgG2 and $\mathrm{IgG} 4$ were recorded (Figure 1). Levels of $\operatorname{IgG1}(\mathrm{r}=$
0.813; $\mathrm{p}<0.001), \operatorname{IgG} 2(\mathrm{r}=0.437 ; \mathrm{p}<0.001), \operatorname{IgG} 3(\mathrm{r}$ $=0.776 ; \mathrm{p}<0.001)$ and $\operatorname{IgM}(\mathrm{r}=0.479 ; \mathrm{p}<0.001)$ correlated significantly with total $P$ falciparum-specific IgG whereas there was no association $(p=0.111)$ between total $\operatorname{IgG}$ and IgG4. 
Table 1: Characteristics of the study participants $(\mathrm{N}=271)$ at delivery from Mutengene, South West Region

\begin{tabular}{|c|c|}
\hline Factor & $\%(\mathbf{n}) *$ \\
\hline \multicolumn{2}{|l|}{$\operatorname{Age}(\text { years })^{\$}$} \\
\hline$\leq 20$ & $31.7(85)$ \\
\hline $21-25$ & $35.5(95)$ \\
\hline$>25$ & $32.8(88)$ \\
\hline \multicolumn{2}{|l|}{ Parity } \\
\hline Primiparae & $33.2(90)$ \\
\hline Secundiparae & $27.7(75)$ \\
\hline Multiparae & 39.1 (106) \\
\hline \multicolumn{2}{|c|}{ IPTp-SP dosage $^{\&}$} \\
\hline 1 dose & $43.1(115)$ \\
\hline$\leq 2$ doses & $46.8(125)$ \\
\hline No IPTp & $10.1(27)$ \\
\hline \multicolumn{2}{|c|}{ \#Prevalence of placental malaria } \\
\hline \multirow[t]{2}{*}{ infection } & 60.9 \\
\hline & $(165)$ \\
\hline \multicolumn{2}{|c|}{ Prevalence of placental malaria } \\
\hline parasitaemia & $37.3(101)$ \\
\hline
\end{tabular}

There was a significant positive correlation among lev- ly with levels of IgG1 and IgG3 whereas IgG4 did not els of IgG1, IgG2 and IgG3. IgM correlated significant- show a relationship with any of the isotypic antibodies measured (Table 2).

Table 2: Correlation between IgM and IgG subtypes specific for crude blood stage $P$. falciparum in parturient Cameroonian women

\begin{tabular}{lcc}
\hline & \multicolumn{2}{c}{ All women } \\
\cline { 2 - 3 } Comparison & $\mathbf{r}_{\text {s }}$ & $\boldsymbol{p}$ \\
IgG1 vs IgG2 & 0.275 & $<0.001$ \\
IgG1 vs IgG3 & 0.652 & $<0.001$ \\
IgG1 vs IgG4 & 0.139 & 0.114 \\
IgG1 vs IgM & $\mathbf{0 . 4 4 0}$ & $<\mathbf{0 . 0 0 1}$ \\
IgG2 vs IgG3 & $\mathbf{0 . 2 8 9}$ & $<\mathbf{0 . 0 0 1}$ \\
IgG2 vs IgG4 & 0.109 & 0.299 \\
IgG2 vs IgM & 0.001 & 0.993 \\
IgG3 vs IgG4 & 0.002 & 0.978 \\
IgG3 vs IgM & $\mathbf{0 . 4 6 1}$ & $<\mathbf{0 . 0 0 1}$ \\
IgG4 vs IgM & 0.120 & 0.175 \\
\hline
\end{tabular}


Isotypic antibody levels, maternal age, parity and IPTp-SP dosage

Age and parity had a significant effect on anti-P falciparum-specific IgG, IgG1, IgG2 and IgG3 levels. Younger ( $\leq 20$ years) mothers and primiparous women had significantly lower mean $\operatorname{IgG} 1, \operatorname{IgG} 2$ and $\operatorname{IgG} 3$ antibody levels compared with older mothers $(>25$ years) and mul- tiparous women (Table 3). Levels of IgG1 and IgG3 were similar between mothers within the 21-25years and older $(>25 y e a r s)$ age groups as well as between secundiparous and multiparous women. Secunduparous women and mothers within 21- 25years age group had significantly lower IgG2 levels when compared with multiparous women and older mothers ( $>$ 25years) respectively (Table 3).

Table 3: Association of mean ( \pm SD) levels of $P$ falciparum-specific isotypic antibodies with maternal age, parity, IPTp-SP dosage and PM infection

\begin{tabular}{|c|c|c|c|c|c|c|c|c|c|c|c|c|}
\hline Factor/Ab isotype & IgG & $p$ & IgG1 & $p$ & IgG2 & ${ }^{\&} p$ & IgG3 & ${ }^{*} p$ & IgG4 & ${ }^{\&} p$ & IgM & ${ }^{\&} p$ \\
\hline \multicolumn{13}{|l|}{ Age(years) } \\
\hline$\leq \mathbf{2 0}$ & $3.46 \pm 0.44$ & 0.080 & $3.45 \pm 0.42$ & 0.003 & $1.43 \pm 0.70$ & 0.027 & $2.71 \pm 0.68$ & 0.009 & $1.49 \pm 0.59$ & 0.060 & $3.08 \pm 0.50$ & 0.646 \\
\hline $21-25$ & $3.51 \pm 0.52$ & 0.566 & $3.53 \pm 0.40$ & 0.120 & $1.45 \pm 0.63$ & 0.022 & $2.88 \pm 0.55$ & 0.263 & $1.27 \pm 0.65$ & 0.756 & $3.22 \pm 0.45$ & 0.042 \\
\hline$>25$ & $3.57 \pm 0.50$ & REF & $3.62 \pm 0.34$ & REF & $1.70 \pm 0.57$ & REF & $2.99 \pm 0.45$ & REF & $1.22 \pm 0.74$ & REF & $3.04 \pm 0.66$ & REF \\
\hline \multicolumn{13}{|l|}{ Parity } \\
\hline Primiparae & $3.43 \pm 0.51$ & 0.024 & $3.46 \pm 0.43$ & 0.007 & $1.40 \pm 0.65$ & 0.008 & $\begin{array}{l}2.67 \pm 0.63 \\
2.88 \pm 0.57\end{array}$ & $\begin{array}{c}<0.001 \\
0.140\end{array}$ & $1.37 \pm 0.64$ & 0.442 & $3.10 \pm 0.48$ & 0.492 \\
\hline Secundiparae & $3.50 \pm 0.46$ & 0.184 & $3.51 \pm 0.39$ & 0.060 & $1.43 \pm 0.64$ & 0.018 & $3.02 \pm 0.47$ & REF & $1.39 \pm 0.62$ & 0.358 & $3.09 \pm 0.56$ & 0.436 \\
\hline Multiparae & $3.59 \pm 0.48$ & REF & $3.62 \pm 0.35$ & REF & $1.70 \pm 0.59$ & REF & & & $1.26 \pm 0.73$ & REF & $3.16 \pm 0.60$ & REF \\
\hline \multicolumn{13}{|l|}{ IPT-SP (doses) } \\
\hline One & $3.60 \pm 0.42$ & 0.006 & $3.60 \pm 0.38$ & 0.031 & $1.56 \pm 0.68$ & 0.911 & $2.89 \pm 0.57$ & 0.201 & $1.38 \pm 0.63$ & 0.155 & $3.18 \pm 0.54$ & 0.043 \\
\hline $\begin{array}{l}\text { Two or more } \\
\text { PM infection }\end{array}$ & $3.41 \pm 0.54$ & REF & $3.49 \pm 0.40$ & REF & $1.55 \pm 0.59$ & REF & $2.79 \pm 0.59$ & REF & $1.20 \pm 0.72$ & REF & $3.03 \pm 0.55$ & REF \\
\hline Positive & $3.56 \pm 0.43$ & 0.234 & $3.58 \pm 0.37$ & 0.06 & $1.56 \pm 0.61$ & 0.413 & $2.89 \pm 0.54$ & 0.65 & $1.48 \pm 0.59$ & $<0.001$ & $3.17 \pm 0.53$ & 0.029 \\
\hline Negative & $3.45 \pm 0.56$ & & $3.47 \pm 0.43$ & & $1.48 \pm 0.67$ & & $2.83 \pm 0.63$ & & $1.07 \pm 0.73$ & & $3.03 \pm 0.56$ & \\
\hline
\end{tabular}

\# defined aspresence of parasites and/or pigment detected by placental blood microscopy and placental histology, Ab = Antibody isotype, PM= Placental malaria, REF= Reference. $P$-values were obtained by use of *Mann-Whitney $\mathrm{U}$ test and ${ }^{\text {\& }}$ Student $\mathrm{t}$ test to compare antibody levels, $\mathrm{P}<0.05=$ significant

Levels of IgG4 and IgM were comparable among age and parity groups. Among women who took IPTp-SP, IgG $(\mathrm{P}=0.006)$ and $\operatorname{IgG1}(\mathrm{P}=0.031)$ antibody levels were significantly lower in mothers who had taken two or more SP doses when compared with levels of those who had a single dose. Conversely, higher $\operatorname{IgM}(\mathrm{P}=0.043)$ levels were seen in mothers who had one dose compared with those who had multiple doses. IgG2, IgG3 and IgG4 levels did not vary with SP dosage (Table 3).

\section{Isotypic antibodies, PM infection and IVS mono- cyte/macrophage infiltration}

PM infection was associated with a statistical significant increase in levels of $\operatorname{IgG} 4(\mathrm{p}<0.001)$ and $\operatorname{IgM}(\mathrm{p}$ $=0.029)$ when compared with levels seen in uninfected mothers (Table 3). Compared to mothers with malaria negative placentas, those with active infection had significantly higher levels of $\operatorname{IgG1}(\mathrm{t}=2.03 ; \mathrm{p}=0.043), \mathrm{IgG} 4$ $(\mathrm{t}=2.62 ; \mathrm{p}=0.01)$ and $\operatorname{IgM}(\mathrm{t}=2.53 ; \mathrm{p}=0.012)$. There were no statistically significant differences between isotypic antibody levels and past infection except for IgG4 where significantly higher $(\mathrm{t}=3.37 ; \mathrm{p}=0.001)$ levels were seen in women with past infection compared with those without infection. Mothers seropositive for IgG4 (37.6\%) were more likely to have placental monocyte/ macrophage infiltration $(\mathrm{OR}=1.83$; 95\% CI: 1.05-3.17; $\mathrm{p}=0.031)$ compared with those seronegative for IgG4 $(24.8 \%)$. No significant differences were observed between other antibody isotypes and presence of placental monocyte/macrophage infiltration. 
Confounding influences on isotypic antibody levels Several exploratory MLR (enter) models were ran to assess the independent factors associated with the levels of antibody isotypes. Since maternal age strongly correlated $(\mathrm{r}=0.781 ; \mathrm{p}<0.001)$ with parity, age was not included in the model. In general, the factors in the models were poor predictors of the different isotypic antibody lev- els to P falciparum crude blood stage antigen (Table 4). Nonetheless, parity, PM infection and IPTp-SP dosage had a significant effect on IgG and IgG1 levels (Table 4). On the other hand, parity significantly affected levels of IgG2 $(\mathrm{P}=0.005)$ and $\operatorname{IgG} 3(\mathrm{P}<0.001)$. PM infection remained associated with IgG4 $(\mathrm{P}=0.002)$ while $\mathrm{PM}$ infection ( $\mathrm{P}=0.014)$ as well as IPTp-SP dosage $(\mathrm{P}=0.043)$ significantly influenced IgM levels (Table 4).

Table 4: Multiple linear regression analyses examining the influence of independent variables on each antibody isotype

\begin{tabular}{cccccc}
\hline $\begin{array}{c}\text { Antibody } \\
\text { isotype }\end{array}$ & $\mathbf{N}$ & Independentvariable & $\boldsymbol{\beta}$-value & P-value & $\mathbf{R}^{2}$ \\
\hline \multirow{2}{*}{ IgG } & 235 & Parity & 0.084 & $\mathbf{0 . 0 2 5}$ & 0.07 \\
& & PM & 0.133 & $\mathbf{0 . 0 4 5}$ & \\
IgG1 & SP dosage & -0.195 & $\mathbf{0 . 0 0 2}$ & 0.08 \\
& 231 & Parity & 0.084 & $\mathbf{0 . 0 0 5}$ & 0.06 \\
IgG2 & PM & -0.153 & $\mathbf{0 . 0 0 3}$ & \\
& & SP dosage & -0.109 & $\mathbf{0 . 0 2 9}$ & \\
IgG3 & Parity & 0.175 & $\mathbf{0 . 0 0 5}$ & \\
& \multirow{2}{*}{50} & PM & -0.146 & 0.610 & \\
& & SP dosage & -0.053 & 0.168 & \\
\end{tabular}

Association of isotypic antibody ratios and PM infection

Different immunoglobulin isotypes can react with the same epitopes and thus in different ways influence the course of an infection. IgG4 may interfere with the protective effect of IgG subtypes (1-3). Thus, we evaluated the ratio of $\operatorname{IgG} 1$ and/or $\mathrm{IgG} 2$ and/or $\mathrm{IgG} 3$ to $\mathrm{IgG} 4$ and/or IgG2 in relation to PM infection status. The mean $\mathrm{IgG} 1+\mathrm{IgG} 3 / \mathrm{IgG} 2+\operatorname{IgG} 4$ and $\mathrm{IgG} 1+\mathrm{IgG} 2+\mathrm{IgG} 3 /$ IgG4 ratios were significantly higher among the uninfected group while higher mean $\operatorname{IgG} 4 / \mathrm{IgG} 2$ ratio was seen in women with PM infection compared with those without infection. There was no statistical difference between placental infection status and mean $\mathrm{IgG} / \mathrm{IgM}$ or $\mathrm{IgM} / \mathrm{IgG}$ ratios (Table 5).

\begin{tabular}{|c|c|c|c|}
\hline \multirow[b]{2}{*}{ Ratio } & \multicolumn{3}{|c|}{ Placental malaria infection ${ }^{\#}$} \\
\hline & PM infected & PM uninfected & $* p$ \\
\hline IgG1/IgG4 & 2.98 & 4.02 & 0.07 \\
\hline IgG2/IgG4 & 1.25 & 1.64 & 0.211 \\
\hline IgG3/IgG4 & 2.39 & 3.27 & 0.074 \\
\hline IgG1 + IgG3/ IgG2 & 5.00 & 5.26 & 0.524 \\
\hline IgG1+ IgG3/IgG2 + IgG4 & 2.42 & 3.47 & 0.009 \\
\hline IgG1 + IgG2 +IgG3/IgG4 & 6.81 & 9.60 & 0.011 \\
\hline IgG1 + IgG2 +IgG3/IgG4 + IgM & 1.80 & 2.02 & 0.073 \\
\hline IgG4/IgG2 & 1.20 & 0.72 & 0.003 \\
\hline IgG/IgM & 1.14 & 1.19 & 0.275 \\
\hline IgM/IgG & 0.90 & 0.90 & 0.323 \\
\hline
\end{tabular}

\# Presence of parasites and/or pigment detected by placental blood microscopy and placental histology, ${ }^{*} P$ values were determined by the non-parametric Kruskal Wallis test; $\mathrm{p}<0.05=$ significant 


\section{Discussion}

This study determined IgG isotypic antibody response pattern to crude P. falciparum blood stage antigen in plasma from Cameroonian parturient women and investigated antibody levels in relation to PM infection. P. falciparum-specific IgG1, IgG4 and IgM levels increased in response to PM infection.

Antibody responses to a crude P. falciparum blood stage extract in parturient women were predominantly cytophilic given the higher levels and prevalence rates of IgG1 and IgG3 subclasses seen relative to $\operatorname{IgG} 2$ and IgG4. These findings are in accordance with reports from previous studies ${ }^{13,15,20}$. Similarly, VSAPAM -specific IgG subclass responses in pregnancy are dominated by IgG1 and IgG3 antibodies as reported by Megnekou et al. ${ }^{13}$ and Eliott et al. ${ }^{15}$. Cytophilic antibodies are usually produced together ${ }^{15}$. In line with this, levels of parasite-specific IgG1 correlated strongly with IgG3 levels. IgG2 is a non-cytophilic antibody and correlated significantly with IgG1 and IgG3 suggesting that in addition to IgG3, IgG2 could also be of major importance in protection against P. falciparum infection. Moreover, parasite-specific IgG2 levels have been reported to increase with age and higher in the older individuals who have progressively developed an efficient protective immunity ${ }^{16,21}$. In line with this, levels of $\operatorname{IgG}, \operatorname{IgG} 1, \operatorname{IgG} 2$ and $\operatorname{IgG} 3$ antibodies significantly increased with age and exposure (measured as antibody reactivity to schizont extract). In conformity with previous reports ${ }^{15}$, IgG4 levels did not correlate with total-IgG nor with subtypes suggesting that IgG4 does not make up a major component of the $\operatorname{IgG}$ response to P. falciparum antigens. Studies have investigated the half-lives of the antibodies directed against merozoite antigens ${ }^{33-34}$. In a Nigerian study, IgG2 and IgG4 responses to EBA (erythrocyte binding antigen) - 175 showed significantly shorter half-lives compared to $\operatorname{IgG1}, \operatorname{IgG} 3$ and total $\mathrm{IgG}$. It is suggested that the shorter half-lives of IgG2 and IgG4 might explain why these subclasses are usually considered to be less important in protection against malaria ${ }^{34}$.

The isotypic antibody distribution pattern observed in the study population was influenced by parity, IPTp-SP dosage and PM infection. This is consistent with recent findings from studies in the same area ${ }^{35}$ and elsewhere ${ }^{36}$. Although parity, IPTp-SP dosage and PM infection were significantly associated with antibody titres, these factors

African Health Sciences Vol 16 Issue 4, December, 2016 were generally poor predictors of isotypic antibody responses to P. falciparum blood stage antigens. Similarly, a study carried out in Yaoundé and Etoa showed that maternal age, parity, asymptomatic parasitemia and drug usage were generally poor predictors of $\mathrm{IgG}$ and subclass-specific responses to non-PAM type VSA ${ }^{13}$. However, our findings confirm previous reports from Cameroon ${ }^{11,12,13}$ and Mozambique 10 showing that levels of IgG antibodies (with the exception of IgG4) against P. falciparum antigen not specifically associated with pregnancy increase with parity. The correlation observed between antibody levels and parity can be explain by the fact that mothers of higher parities were older in age and consequently may produce higher levels of $\mathrm{IgG}$ antibodies due to age-dependent immunity. The role of age-associated anti-parasite immunity in limiting P. falciparum infection among multigravid women in areas of high transmission has been suggested ${ }^{37}$. A study in Uganda showed that naturally acquired immunity to serine repeat antigen-5 (SE36) and merozoite surface protein-1 (MSP119 and MSP142) in pregnant women were associated with reduced placental parasitaemia ${ }^{23}$.

The recent implementation of IPTp in the mt Cameroon area has undoubtedly improved antimalarial protection, reducing placental malaria parasitaemia ${ }^{38}$. Conversely, a successful regime of IPTp could decrease exposure to malaria during pregnancy and antibody titres to key malarial antigens could decline ${ }^{2}$. In line with this, lower levels of $\mathrm{IgG}, \mathrm{IgG} 1$ and $\mathrm{IgM}$ in response to P. falciparum antigens were observed in mothers who took two or more SP doses. Moreover, we previously showed that women on two or more SP doses were less likely to be infected ${ }^{24}$. The impact of IPTp-SP on maternal immunity and on foetal transplacental immunity in malaria endemic regions has important implication on the infant's susceptibility to malaria early in life. Interestingly, a one -year follow-up study of infants from birth in the same study area showed that infants born to mothers on two or more doses of IPTp-SP were more vulnerable to clinical episodes ${ }^{35}$. This reduced protection of these infants to malaria may be attributed to lower IgM levels in these mothers and their 1-year-old infants which suggest that the reduced immunity may be linked to the reduced maternal exposure to the parasite during pregnancy. It is possible that IPTp might affect infant immune response by reducing levels of transplacental passage of maternal antibodies ${ }^{39}$ or decrease in utero sensitisation to malarial antigens ${ }^{40-41}$. 
Plasma from parturient women with and without PM infection revealed that infection during pregnancy boosts antibody responses where higher titres of $\operatorname{IgG1}$, IgG4 and IgM to crude blood stage antigen were associated with placental malaria parasitaemia infection. Studies in Mozambique $^{10}$, Malawi ${ }^{15}$ and Saudi ${ }^{42}$ have also reported higher levels of specific IgG to variant surface antigens in association with PM infection. Increased maternal antibody levels reflect past or current exposure to P. falciparum prior to delivery, consistent with previous suggestions $^{10,13}$.

To the best of our knowledge, we report here for the first time an association between levels of anti-malarial specific IgG4 and PM infection in parturient women. On the contrary,exposure to placental malaria was not associated with induction of VSAPAM-specific IgG4 subtype in a previous study ${ }^{15}$. It has been hypothesised that the profile of IgG subclass response induced during infection may differ mainly with the type of antigen and possibly polymorphisms in antigens ${ }^{43-44}$. Findings of this study showed higher IgG4/IgG2 ratios observed in women with infection. Aucan et al. ${ }^{21}$ observed that the positive correlation of IgG4 with the risk of infection was stronger when IgG2 levels were low. More so, a recent study in Saudi investigated the patterns of antibody isotype in relation to relative risk of malaria infection in pregnant women and observed significantly higher levels anti-malarial $\mathrm{IgG}$, IgG2 and IgG4 in women with asymptomatic malaria infection compared with those without infection ${ }^{42}$.

\section{Acknowledgements}

We thank the entire staff of the Mutengene Medical Centre and the mothers who volunteered to participate in this study. We are grateful to Mr Epie and all the midwives in particular for cooperation and support in this research work. Our profound gratitude to the Jean-Baptist Bengono and the entire staff of the Anatomy and Pathology Laboratory (ANAPATH), University of Yaoundé teaching hospital (CHU), Cameroon and the Department of Immunology, University of Stockholm for their technical assistance. This investigation received financial support from the Swedish Institute, Stockholm Guest Scholarship to A. J. K.

\section{Conflict of interest}

PM infection and IPTp-SP dosage influenced isotypic antibody responses to P. falciparum blood stage antigens.
IgG4 levels increased in response to PM infection and thus its role in susceptibility to PM infection warrants further investigation.

\section{Acknowledgements}

We thank the entire staff of the Mutengene Medical Centre and the mothers who volunteered to participate in this study. We are grateful to Mr Epie and all the midwives in particular for cooperation and support in this research work. Our profound gratitude to the Jean-Baptist Bengono and the entire staff of the Anatomy and Pathology Laboratory (ANAPATH), University of Yaoundé teaching hospital (CHU), Cameroon and the Department of Immunology, University of Stockholm for their technical assistance. This investigation received financial support from the Swedish Institute, Stockholm [Guest Scholarship to A. J. K.]

\section{References}

1. Brabin BJ. Epidemiology of infection in pregnancy. Rev Infect Dis 1985; 7:579-603.

2. Rogerson SJ, Hviid L, Duffy PE, Leke R, Taylor DW. Malaria in pregnancy: pathogenesis and immunity. Lancet 2007; 7:105-117.

3. Brabin BJ, Romagosa C, Abdelgalila S, et al. The sick placenta: the role of malaria. Placenta 2004; 25:359-378. 4. Fried M, Nosten F, Brockman A, Brabin BJ, Duffy PE. Maternal antibodies block malaria. Nature 1998; 395:851852.

5. Ricke CH, Staalsoe T, Koram K, et al. Plasma antibodies from malaria exposed pregnant women recognize variant surface antigens on Plasmodium falciparum-infected erythrocytes in a parity-dependent manner and block parasite adhesion to chondroitin sulfate A. J Immunol 2000; 165:3309-16.

6. Staalsoe T, Shulman CE, Bulmer JN, Kawuondo K, Marsh K and Hviid L.. Variant surface antigen-specific $\mathrm{IgG}$ and protection against clinical consequences of pregnancy-associated Plasmodium falciparum malaria. Lancet 2004; 363:283-289.

7. Duffy PE, Fried M. Antibodies that inhibit Plasmodium falciparum adhesion to chondroitin sulfate $\mathrm{A}$ are associated with increased birth weight and the gestational age of newborns. Infect Immun 2003; 71:6620-6623.

8. Desai M, Ter Kuile FO, Noston F, McGready R, Asamoa $\mathrm{K}$, et al. Epidemiology and burden of malaria in pregnancy. Lancet Infect Dis 2007; 7:93-104.

9. Rovira-Vallbona E, Doban C, Bardaj A, , et al. Tran-

African Health Sciences Vol 16 Issue 4, December, 2016 
scription of var Genes other than var2csa in Plasmodium falciparum parasites infecting Mozambican pregnant women. J Infect Dis. 2011; 204:27-35.

10. Mayor A, Rovira-Vallbona E, Machevo S, et al. Parity and placental infection affect antibody responses against Plasmodium falciparum during Pregnancy. Infect Immun. 2011; 79 (4):1654-1659.

11. Mvondo JL, James MA, Sulzer AJ andCampbell CC. Malaria and pregnancy in Cameroonian women. Naturally acquired antibody responses to asexual blood-stage antigens and the circumsporozoite protein of Plasmodium falciparum. Trans R Soc Trop Med Hyg. 1992; 86:486-490. 12. Fievet N, Cot M, Ringwald P, Bickii J, Dubois B, et al. Immune response to Plasmodium falciparum antigens in Cameroonian primigravidae: evolution after delivery and during second pregnancy. Clin Exp Immunol. 1997;107:462-467.

13. Megnekou R, Staalsoe T, Taylor DW, Leke R andHviid L. Effects of pregnancy and intensity of Plasmodium falciparum transmission on Immunoglobulin G Subclass responses to variant surface Antigens. Infect Immun. 2005; 73(7):4112-4118.

14. Piper KP, Roberts DJ andDay KP. Plasmodium falciparum: analysis of the antibody specificity to the surface of the trophozoite-infected erythrocyte. Exp Parasitol.1999; 91:161-169.

15. Elliott SR, Brennan AK, Beeson JG, et al. Placental malaria induces variant-specific antibodies of the cytophilic subtypes immunoglobulin G1 (IgG1) and IgG3 that correlate with adhesion inhibitory activity. Infect Immun. 2005; 73: 5903-5907.

16. Cabrera G, Yone C, Tebo AE, Van Aaken J, Lell B, et al. Immunoglobulin $G$ isotype responses to variant surface antigens of Plasmodium falciparum in healthy Gabonese adults and children during and after successive malaria attacks. Infect Immun. 2004; 72:284-294.

17. Yone CL, Kremsner PG and Luty AJ. Immunoglobulin $G$ isotype responses to erythrocyte surface-expressed variant antigens of Plasmodium falciparum predict protection from malaria in African children. Infect Immun. 2005; 73:2281-2287.

18. Shi YP, Sayed U, Qari SH, Roberts JM, Udhayakumar $\mathrm{V}$, et al. Natural immune response to the C-terminal 19kilo dalton domain of Plasmodium falciparum merozoite surface protein 1. Infect Immun 1996; 4:2716-2723.

19.Day KP, Marsh K. Naturally acquired immunity to Plasmodium falciparum. Immunol Today 1991; 12:68-71. 20. Deloron P, Dubois B, Le Hesran JY, Riche D, Fievet, et al. Isotypic analysis of maternally transmitted Plasmodium falciparum-specific antibodies in Cameroon, and relationship with risk of P. falciparum infection. Clin Exp Immunol. 1997; 110:212-218.

21. Aucan C, Traoré Y, Tall F, Nacro B Traoré-Leroux T, et al. Malaria high Immunoglobulin G2 (IgG2) and low IgG4 levels are associated with human resistance to Plasmodium falciparum. Infect Immun 2000; 68(3):1252.

22. Owalla TJ, Palacpac NMQ, Horii HST, Egwang, TG. Association of naturally acquired $\operatorname{IgG}$ antibodies against Plasmodium falciparum serine repeat antigen-5 with reduced placental parasitemia and normal birth weight in pregnant Ugandan women: A pilot study. Parasitology International 2013; 62(3): 37-239.

23. Achidi EA, Minang JT, Anchang JK, Ahmadou MJ, Troye-Blomberg M. Plasmodium falciparum inhibitory capacities of paired maternal-cord sera from South-West Province, Cameroon. J Trop Pediatrics 2004; 51(3):182-90. 24. Anchang-Kimbi JK, Achidi EA, Nkegoum B, Sverremark-Ekstrom E, Troye-Blomberg M. Diagnostic comparison of malaria infection in peripheral blood, placental blood and placental biopsies in Cameroonian parturient women. Malar J 2009; 8:126.

25. WHO. A strategic framework for malaria prevention and control during pregnancy in the African region. AFR/MAL/report 2004.

26. Romagosa C, Menendez C, Ismail MR, Quintó L, Ferrer B, et al. Polarisation microscopy increases the sensitivity of haemozoin and Plasmodium detection in the histological assessment of placental malaria Acta Tropica 2004; 90:277-284.

27. Ismail MR, Ordi J, Menendez C, Ventura PJ, Aponte JJ, et al. Placental pathology in malaria: a histological, immunohistochemical, and quantitative study. Human Pathology, 2000; 31: 85-93.

28. Rogerson SJ, Pollina E, Getachew A, Tadesse E, Lema $\mathrm{VM}$, et al. Placental monocyte infiltrates in response to Plasmodium falciparum infection and their association with adverse pregnancy outcomes. Am J Trop Med Hyg 2003; 68:115-19.

29. Troye-Blomberg M, Perlmann H, Patarroyo ME, Perlmann P. Antigen regulation of the immune response in P. falciparummalaria. II. Antigen specific proliferative responses in vitro. Clin Exp Immun 1983; 53:345-9.

30. Perlmann H, Perlmann P, Berzins K, Wahlin B, TroyeBlomberg M, et al. Dissection of the human antibody response to the malaria antigen Pf155/RESA into epitope specific components. Immunol Rev 1989; 112:115-31. 
31. Farouk SE, Dolo A, Bereczky S, Kouriba B, Maiga B et al. Different antibody- and cytokine-mediated responses to Plasmodium falciparum parasite in two sympatric ethnic tribes living in Mali. Micro Infect 2005; 7(1):110-7. 32. Fernandez V. Separation of Plasmodium falciparum mature stages in percoll/sorbitol gradients. In: Ljungstrom I, Perlmann H, Schlichtherie M, Scherf A, Wahlgren M. (eds), Methods in Malaria Research. MR4/ATCC 2004; Pp 26-27.

33. Kinyanjui SM, Conway DJ, Lanar DE and Marsh K. IgG antibody responses to Plasmodium falciparum merozoite antigens in Kenyan children have a short halflife. Malaria Journal 2007, 6:82.

34. Ahmed Ismail H, Tijani M K, Langer C, Reiling L,
White M T, Beeson JG, Wahlgren M, Nwuba R and Persson KEM. Subclass responses and their half-lives for antibodies against EBA175 and PfRh2 in naturally acquired immunity against Plasmodium falciparum malaria. Malaria Journal 2014, 13:425..

35 .Apinjoh T.O, Anchang-Kimbi J.K, Mugri, R N -Yafi, CN Tata, RB. Chi, HF Tangoh, DA . Loh, BT and. Achidi, EA.Determinants of Infant Susceptibility to Malaria During the First Year of Life in South Western Cameroon. Open Forum Infectious Diseases 2015; DOI: 10.1093/ ofid/ofv012.

36. Aitken EH, Mbewe B, Luntamo M, Kulmala T, Beeson JG et al. Antibody to P. falciparum in Pregnancy Varies with Intermittent Preventive Treatment Regime and Bed Net Use. PLoS one 